\title{
Multiscale Boundary Identification for Medical Images
}

\author{
ZuYing Wang, Mrinal Mandal, and Zoltan Koles \\ Department of Electrical and Computer Engineering, \\ University of Alberta, Edmonton, AB, Canada \\ \{zywang, mandal\}@ece.ualberta.ca, z.koles@ualberta.ca
}

\begin{abstract}
Boundary identification in medical images plays a crucial role in helping physicians in patient diagnosis. Manual identification of object boundaries is a time-consuming task and is subject to operator variability. Fully automatic procedures are still far from satisfactory in most real situations. In this paper, we propose a boundary identification method based on multiscale technique. Experimental results have shown that the proposed method provides superior performance in medical image segmentation.
\end{abstract}

Keywords: Boundary identification, multiscale edge detection.

\section{Introduction}

Various medical imaging modalities such as the radiograph, computed tomography (CT), and magnetic resonance (MR) imaging are widely used in routine clinical practice. Boundary identification (BI) of deformed tissue plays a crucial role in accurate patient diagnosis. For example, an MR brain image can be segmented into different tissue classes, such as gray matter, white matter, and cerebrospinal fluid. Unfortunately, manual BI methods are very time consuming and are often subjective in nature. Recently an edge-based MR image segmentation method, mtrack [1], has been proposed. This algorithm provides a reasonably good performance. However, the edge-based segmentation algorithm has some limitations, such as sensitivity to noise and presence of gaps in detected boundaries. Together, these effects degrade the quality of the detected boundaries. The discrete wavelet transform (DWT) has recently been shown to be a powerful tool in multiscale edge detection. Marr and Hildreth [2] introduced the concept of multiscale edge detection for detecting the boundaries of objects in an image. Mallat and Zhong [3] showed that multiscale edge detection can be implemented by smoothing a signal with a convolution kernel at various scales, and detecting sharp variation points as edges. Tang et al. [4] studied the characterization of edges with wavelet transform and Lipschitz exponents.

In this paper, we propose a multiscale boundary identification algorithm, based on DWT, for better edge-based segmentation of MR images. The remainder of this paper is organized as follows. Section 2 briefly reviews the related background work. Section 3 presents the proposed method developed based on

A. Ghosh, R.K. De, and S.K. Pal (Eds.): PReMI 2007, LNCS 4815, pp. $177185,2007$.

(C) Springer-Verlag Berlin Heidelberg 2007 
the existing theory for multiscale edge detection and boundary identification. Section 4 presents the performance evaluation of the proposed method. The conclusions are presented in Section 5.

\section{Review of Related Work}

In this section, we present a brief review of the related background work.

\section{1 mtrack Algorithm}

The mtrack method [1] has been developed at the University of Alberta for medical image segmentation, especially for MR images of the head. As shown in Fig. 1, it includes three major modules: edge detection, edge feature extraction, and edge tracing. The three modules are explained below.

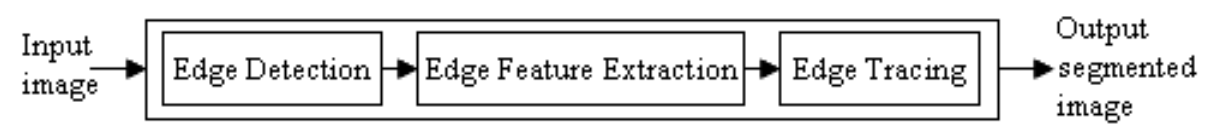

Fig. 1. Block diagram of mtrack algorithm

1. Edge detection: In this module, the edge of an image is obtained using a Canny edge detector. The detector is applied for six operator directions. Subpixel edge resolution is obtained by linearly interpolating the zero crossings in the second derivative of a Gaussian filtered image.

2. Edge Feature Extraction: Edge features are extracted from the detected edges. For each edge point along the operator direction, the top and bottom intensity points of that edge slope are obtained by examining the 1st and 2nd derivatives curves. The coordinates of the subpixel edge points and the top and bottom intensity on each side of the edge points are combined as four-dimensional edge information.

3. Edge Tracing: A target tracking algorithm is used to link the edge points to form an object boundary. The edge information is assumed to be position information of a hypothetical object (target) that moves along the boundary. Target tracking starts from a starting point, follows the path of the target (edge) until no further edge point can be founded on the track or the starting point is revisited. Thus, the boundary can be drawn by linking all the edge points that are found in the path of the target, including the starting point.

The mtrack software can perform automatic tracking and can also manually cut and link tracks to form the desired contours. However, some problems remain: First, the tracking performance depends on the starting point. Secondly, it depends on the track direction. The tracking algorithm also involves the possibility of self-intersection, the selection of appropriate parameter values, and the possibility that a closed contour is not formed in all cases. The main limitation of this algorithm is that it is sensitive to noise. 


\subsection{Multiscale Edge Detection Technique}

In this paper, we use the multiscale edge detection technique proposed in [3]. We present a brief review of Mallat's edge detection technique in this section. The schematic of multiscale edge detection is shown in Fig. 2. Consider a wavelet function $\Psi_{s}(x, y)$ at scale $s$. The 2-D wavelet-decomposed image $f(x, y)$ at scale $s$ has two components: $W_{s}^{1}(x, y)=f * \Psi_{s}^{1}(x, y)$ and $W_{s}^{2}(x, y)=f * \Psi_{s}^{2}(x, y)$ where $\Psi_{s}^{1}(x, y)$ and $\Psi_{s}^{2}(x, y)$ are, respectively, horizontal and vertical filters. At each scale $s$, the modulus of the gradient vector is called the wavelet transform modulus, and is defined as

$$
M_{s}(x, y)=\sqrt{\left|W_{s}^{1}(x, y)\right|^{2}+\left|W_{s}^{2}(x, y)\right|^{2}} .
$$

The angle of the wavelet gradient vector for each scale is given by

$$
A_{s}(x, y)=\arctan \left(W_{s}^{2}(x, y) / W_{s}^{1}(x, y)\right) .
$$

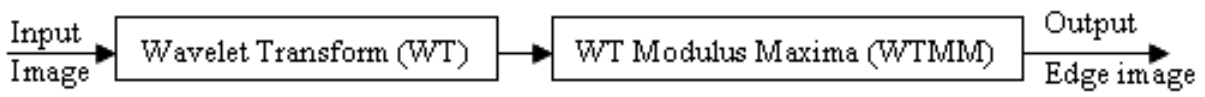

Fig. 2. Block diagram of multiscale edge detection [3]

For a 2-D image, the wavelet transform modulus maxima (WTMM) are located at points where the wavelet transform modulus is larger than its two neighbors in one-dimensional neighborhoods along the angle direction. The local regularity of a signal is characterized by the decay of the wavelet transform across scales [3], and it can be measured in terms of Lipschitz exponent. For a signal $f(x)$, the Lipschitz exponent $\alpha$ satisfies the following:

$$
\left|W_{s}(x)\right| \leq A s^{\alpha}
$$

where $W_{s}(x)$ are wavelet coefficients at scale $s$, and $A$ is a positive constant. Eq. (3) states that the modulus of the wavelet coefficients of a signal vary with the scale $s$ according to the Lipschitz regularity of that signal. The characterization of edges by Lipschitz exponent and wavelet transform has been investigated by Mallat et al. [3]. It has been shown that positive Lipschitz exponent $\alpha$ corresponds to edge structure (i.e. step edge) and negative Lipschitz exponent $\alpha$ corresponds to Dirac structure (i.e. spike noise).

\section{Proposed Method}

In this section, we present the proposed multiscale BI method. The proposed method is developed based on the classical wavelet theory for multiscale edge detection and BI. It has been evaluated in the mtrack framework to see if the 
proposed method is useful for edge-based BI. The schematic of the integrated method is shown in Fig. 3. A comparison between Fig. 3 and Fig. 1 reveals that the proposed method replaces Canny edge detection with multiscale edge detection, and adds multiscale boundary identification before edge tracing. The two new modules in Fig. 3 are explained below.

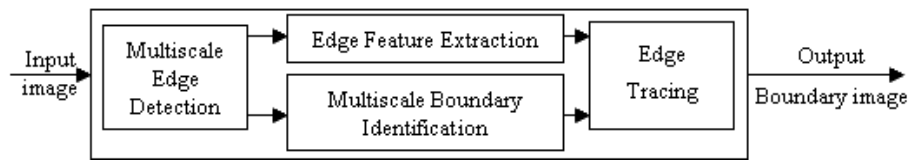

Fig. 3. Block diagram of the proposed boundary identification algorithm

\subsection{Multiscale Edge Detection}

The multiscale edge detection algorithm proposed by Mallat et al. [3] was reviewed in section 2.2. In this paper, we use this algorithm to calculate multiscale edges. The schematic of the proposed multiscale edge detection is shown in Fig. 4. A comparison between Fig. 4 and Fig. 2 reveals that the proposed multiscale edge detection adds two new modules to improve the edge detection performance after traditional edge detection technique. These new modules are explained in the following discussion.



Fig. 4. Block diagram of proposed multiscale edge detection

We can determine the WTMM for each scale of a MR image. The WTMM points at each decomposition level are shown in Fig. 5 as white pixels in the binary image. It is shown in Fig. 5 that when the scale increases, the details and the noise effect decrease quickly. As a result, the edge locations may change in higher scales. We use the WTMM of the first scale $s_{1}$ (i.e., the highest resolution) as the edge image. By looking at the WTMM at different scales, we find that edges of higher significance (i.e., stronger edges) are more likely to be kept by the wavelet transform across scales. Edges of lower significance (i.e., weaker edges) are more likely to disappear at higher scales.

In theory, WTMM can be found along the gradient angle. But practically, we found that only using one-dimensional neighborhood along the angle direction to decide an edge pixel is not sufficient, because a pixel can be part of one edge in one angle direction and be part of another edge in another angle direction. Therefore, we check all the eight pixels around one pixel in the centre for four different directions to decide whether the centre pixel is a WTMM in 


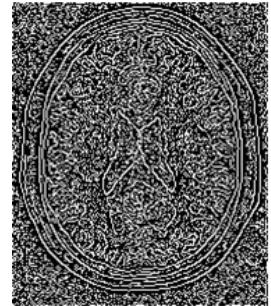

(a)

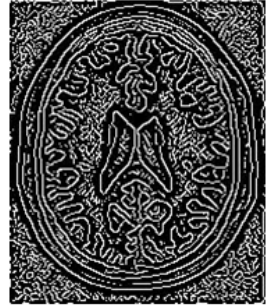

(b)

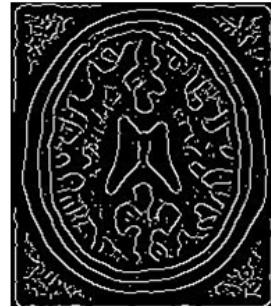

(c)

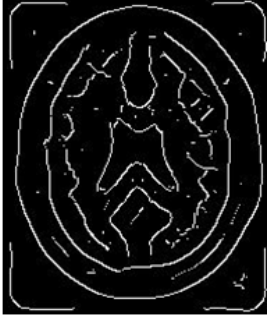

(d)

Fig. 5. WTMM at four scales: a) $s_{1}$; b) $s_{2}$; c) $s_{3}$; d) $s_{4}$

each direction. In order to achieve more accurate edge pixel location, subpixel edge coordinates need to be obtained. We can interpolate the edge pixel that we found in the WTMM process, and fit the pixel coordinates in a polynomial curve to get subpixel resolution. If the pixel is the local maximum, then we record it as an edge pixel in that direction.

\subsection{Multiscale Boundary Identification}

The schematic of the multiscale boundary identification is shown in Fig. 6. We present each module in the following sections.

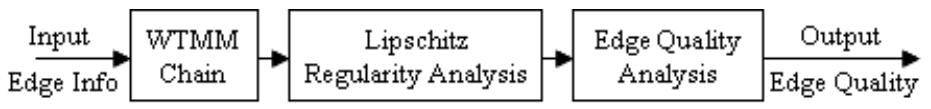

Fig. 6. Block diagram of multiscale boundary identification

2-D WTMM Chain: After we obtain the WTMM for each scale, we need to chain those maxima across scales to indicate where the true edge is. If it is the true edge, it should not disappear during the wavelet decay. We start the WTMM chaining process from the highest scale down to the lowest scale. We need to link the maxima points between two adjacent scales and record the WTMM chain.

We do a four-level decomposition and have four scales with WTMM found for each scale. We start at the highest scale (i.e., scale $s_{4}$ ), and for each maxima pixel, we search in the previous scale (i.e., scale $s_{3}$ ) within a small region. Then a qualification test is performed to check if there is one maxima pixel among all these pixels in the window that has strongest connection to the considered pixel. We link these two maxima pixels together. It is possible that we may find two pixels in scale $j$ that could connect to the same maxima pixel in scale $j-1$. However, we would like to have a one-to-one match to the lower scale maxima pixel for this chaining process. In order to prevent this competing situation, an 
optimization method [5] is used to optimally perform one-to-one maxima pixel matching. A WTMM chaining record is maintained, in which a higher score is assigned to a stronger edge pixel that survives along the wavelet decay, whereas a lower score is assigned to a weaker edge pixel. We define our 2-D WTMM chaining record by 'Scale Depth', as it demonstrates how deep the WTMM pixel is connected through scales.

Lipschitz Regularity Analysis: The Lipschitz exponent can be used to distinguish step and spike edges. However, the Lipschitz exponent is controlled by the log of wavelet modulus across scales. Therefore, we have used the wavelet modulus across scales to identify the boundaries.

Edge Quality Analysis: In order to increase the robustness of the detected edges, we carry out an edge quality test. Here, the amplitude and slope information of the WTMM chain through scales are used to give the strength of each point. A fuzzy logic system is used to determine if a detected edge is a strong edge or a weak edge.

\section{Experimental Setup and Results}

The performance of the proposed technique has been evaluated using the MR images obtained from Montreal Neurological Institute (MNI) [6]. The data is obtained as $1 \mathrm{~mm}$ slice thickness, with pixels per slice, and 8 bits per pixel resolution. The slices are oriented in the transverse plane. There are total 181 slices. We have used the MNI data with $1 \%, 3 \%, 5 \%, 7 \%$ and $9 \%$ noise, and $0 \%, 20 \%$ non-uniformity to evaluate performance. We use MNI slice 95 with $3 \%$ of noise and $20 \%$ of non-uniformity to show an example of evaluation. The comparisons of MR image edge tracing results are presented to demonstrate that the multiscale boundary identification method improves the edge tracing performance.

\subsection{Evaluation Criteria}

To evaluate the performance, we compare the detected boundary with the true boundary. Let $(m, n)$ represent coordinate of a point, and $b(m, n)$ is the boundary image, i.e., $b(m, n)=1$ if $(m, n)$ is a true boundary point. Let the output tracked boundary image be denoted by $t(m, n) . t(m, n)=1$ represents the detected boundary points. We define three criteria as follows:

(1) The total number of track points $N_{T P}$ on the detected boundary image is obtained as: $N_{T P}=\sum_{m, n} t(m, n)$.

(2) The total number of good points $N_{G P}$ on the detected boundary image is obtained as: $N_{G P}=\sum_{m, n} t(m, n) \cdot b(m, n)$.

(3) The track point ratio $R$ is defined as: $R=N_{G P} / N_{T P}$.

Ideally, track point ratio $\mathrm{R}$ should be 1 . However, because the edge tracing algorithm can trace on false boundary points, $\mathrm{R}$ is normally less than 1 . The higher track point ratio corresponds to the better tracing result. Therefore, we 
Table 1. Four evaluation methods. MED: Multiscale Edge Detection; ET: Edge Tracing; MBI: Multiscale Boundary Identification, EFE: Edge Feature Extraction.

\begin{tabular}{|c|c|}
\hline Method 1 & MED+ET \\
Method 2 & MED+MBI+ET \\
Method 3 & MED+EFE+ET \\
Method 4 & MED+EFE/MBI+ET \\
\hline
\end{tabular}

use the track point ratio $\mathrm{R}$ to represent the performance of the output boundary image obtained. For each input image, four different testing methods for the edge tracing algorithm are used for the evaluation comparison. Table 1 shows the modules of the four methods. We have tested all four methods to evaluate the degree of improvement obtained using the multiscale boundary identification method. Note that Method 4 is the proposed BI method shown in Fig. 3.

\subsection{Performance}

Fig. 7 shows a gray/white boundary tracking result by four methods. The four methods use the same edge image obtained from multiscale edge detection, and the starting point chosen for edge tracing is in the same location of the edge image. It is observed that Methods 3 and 4 provide much better performance compared to Methods 1 and 2. Between Fig. 7 (c) and (d), we observe that the small boundaries tracked in the left-bottom quarter in Fig. 7 (d) are more accurate than in Fig. 7 (c). Therefore, Method 4 is better than Method 3.

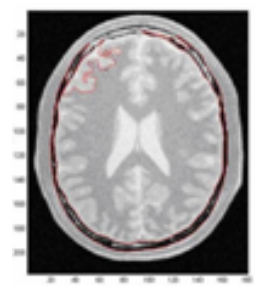

(a)

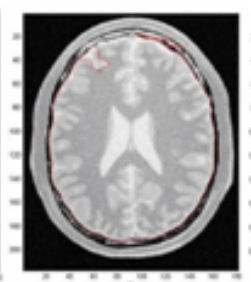

(b)

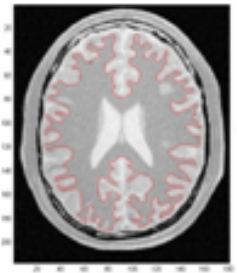

(c)



(d)

Fig. 7. Gray/white boundary tracks for test MR image. (a) Method 1; (b) Method 2; (c) Method 3; (d) Method 4.

Table 2 lists the statistical results of 30 tracks of test image for each method. The mean $(\mu)$, median $(\eta)$, and standard deviation $(\sigma)$ for $N_{G P}, N_{T P}$, and $R$ are calculated. It is again observed that Method 4 provides the best edge tracing result than others. Also note that the standard deviation in the last row is the lowest among others ( $\sigma_{R}$ of Method 4 is five times less than Method 3$)$. This means that the edge tracing using Method 4 is less dependent of the choice of the starting point. The performance evaluation is carried out with $1 \%, 3 \%, 5 \%, 7 \%$ 
Table 2. Statistical results of 30 tracks

\begin{tabular}{|c||c|c|c||c|c|c||c|c|c|}
\hline \multicolumn{1}{|c||}{ Method } & \multicolumn{3}{c||}{$N_{G P}$} & \multicolumn{3}{c||}{$N_{T P}$} & \multicolumn{3}{c|}{$R$} \\
\cline { 2 - 8 } & $\eta_{G P}$ & $\mu_{G P}$ & $\sigma_{G P}$ & $\eta_{T P}$ & $\mu_{T P}$ & $\sigma_{T P}$ & $\eta_{R}$ & $\mu_{R}$ & $\sigma_{R}$ \\
\hline \hline 1 & 201 & 209.4 & 132.8 & 2032.5 & 1813.2 & 821.4 & 0.126 & 0.155 & 0.141 \\
2 & 158.5 & 137.9 & 68.6 & 590 & 533.2 & 217.7 & 0.255 & 0.283 & 0.127 \\
3 & 1024 & 1007.8 & 449.1 & 1623 & 1352.4 & 580.4 & 0.745 & 0.704 & 0.103 \\
4 & 1234.5 & 1144.6 & 249.3 & 1609.5 & 1500.4 & 308.9 & 0.765 & 0.759 & 0.025 \\
\hline
\end{tabular}

Table 3. $\mathrm{R}$ of MNI data slice 95 with $20 \%$ non-uniformity

\begin{tabular}{|c||c|c|c||c|c|c|}
\hline \multicolumn{1}{|c||}{ Noise Level } & \multicolumn{3}{c||}{ Method3 } & \multicolumn{3}{c|}{ Method4 } \\
\cline { 2 - 7 } & $\eta_{R}$ & $\mu_{R}$ & $\sigma_{R}$ & $\eta_{R}$ & $\mu_{R}$ & $\sigma_{R}$ \\
\hline \hline $1 \%$ & 0.836 & 0.823 & 0.029 & $\mathbf{0 . 8 1 7}$ & $\mathbf{0 . 8 1 9}$ & $\mathbf{0 . 0 1 1}$ \\
$3 \%$ & 0.745 & 0.704 & 0.103 & $\mathbf{0 . 7 6 5}$ & $\mathbf{0 . 7 5 9}$ & $\mathbf{0 . 0 2 5}$ \\
$5 \%$ & 0.356 & 0.360 & 0.144 & $\mathbf{0 . 5 8 1}$ & $\mathbf{0 . 5 7 3}$ & $\mathbf{0 . 0 4 9}$ \\
$7 \%$ & 0.255 & 0.260 & 0.117 & $\mathbf{0 . 4 3 0}$ & $\mathbf{0 . 4 1 9}$ & $\mathbf{0 . 0 4 7}$ \\
$9 \%$ & 0.238 & 0.224 & 0.095 & $\mathbf{0 . 3 0 5}$ & $\mathbf{0 . 3 0 3}$ & $\mathbf{0 . 0 6 8}$ \\
\hline
\end{tabular}

and $9 \%$ of noise levels, and $0 \%, 20 \%$ non-uniformity levels in the synthetic MNI data. Table 3 lists the track point ratio of Method 3 and Method 4 on various noise levels $(1 \%, 3 \%, 5 \%, 7 \%$ and $9 \%)$ with $20 \%$ non-uniformity. It is observed that as the noise level increases, the track point ratio of both methods decreases. At each noise level, Method 4 performs better than Method 3, as it has higher $\mu_{R}$ and $\eta_{R}$ values and less $\sigma_{R}$ value, which means the edge tracing by Method 4 is more accurate and less dependent on the starting point. The same trend has also been seen for $0 \%$ non-uniformity. As seen in the experiments, the edge tracing using Method 4 performs better than Method 3. This means that the multiscale boundary identification added into the edge tracing algorithm more accurately tracks the gray/white boundary in the synthetic MR images. Using edge quality from multiscale boundary identification, makes the edge tracing less sensitive to noise, reduces tracing of false edges, and most importantly, the edge tracing is less dependent on the choice of starting point. More simulation results with different data sets (i.e. real MR images) provide a similar trend in the performance [7].

\section{Conclusion}

In this paper, we propose a robust multiscale boundary identification technique for medical images. We enhance the traditional wavelet-based edge detection technique by obtaining directional edges with subpixel resolution. For multiscale boundary identification, we obtain the scale depth of the edge through WTMM chain. We further separate the true edge from noise by Lipschitz analysis, and we obtain the quality for each edge point by fuzzy logic. Experiments results show that the boundary identification preformed by multiscale edge quality analysis 
improves the accuracy of the edge tracing significantly, and the tracking is less dependent on the choice of starting point.

\section{References}

1. Withey, D.: Dynamic edge tracing: recursive methods for medical image segmentation, Ph.D. Thesis, University of Alberta, Canada (2006)

2. Marr, D., Hildreth, E.: Theory of edge detection. Proc. of the Royal Society of London 207, 187-217 (1980)

3. Mallat, S., Zhong, S.: Characterization of signals from multiscale edges. IEEE Trans. on PAMI 14(7), 710-732 (1992)

4. Tang, Y.Y., Yang, L., Feng, L.: Characterization and detection of edges by Lipschitz exponents and MASW wavelet transform. Proc. Intl. Conf. on Pattern Recognition 2, 1572-1574 (1998)

5. Bertsekas, D.P., Castanon, D.A.: A forward/reverse auction algorithm for asymmetric assignment problems. Computational Optimization and Applications 1, 277-297 (1992)

6. MNI data set (August 2005), http://www.bic.mni.mcgill.ca/brainweb/

7. Wang, Z.Y.: Multiscale Boundary Identification for Medical Images. M.Sc. Thesis, University of Alberta, Canada (2007) 